\title{
Acción del óleo calcáreo en el tratamiento de las queilitis por contacto crónicas.
}

Calcareous action of oil in the treatment

of chronic contact cheilitis.
Óleo calcário em ação no tratamento

da crônica queilite.
Fecha de Recepción

21 de marzo de 2013
Aceptado para su publicación

26 de junio de 2013
Víctor Ricardo Fernández

Roque Oscar Rosende

María Mercedes González

Elena Soledad Ortiz Barreto

Rafael Arnaldo Vallejos

\section{Resumen}

Las queilitis por contacto crónicas son patologías con una alta incidencia, que cursa con escasa sintomatología, siendo sus probables transformaciones los motivos por la cual merezca ser atendida.

El proceso es una reacción inflamatoria inespecífica a la acción de agentes exógenos físicos como la luz actínica, los rayos ultravioletas, y químicas como cosméticos, alimentos, galatos, dentífricos, cepillos dentales, metales, prótesis dentales y plantas. La incidencia es mayor en el labio inferior y preferentemente en el sexo femenino.

El linimento óleo calcáreo ha demostrado ser de utilidad en el tratamiento sintomático de las queilitis por contacto agudas. El presente trabajo busca evaluar la acción terapéutica del linimento óleo calcáreo en el tratamiento de las queilitis por contacto crónicas.

\section{Palabras Clave}

Inflamación - labio - queilitis - tratamiento farmacológico - citología.

\section{Summary}

The cheilitis for contact chronicles is pathologies with a high incidence that studies with scarce symptomatology, being their probable transformations the reasons for which deserves to be assisted.

The process is a reaction inflammatory unspecific to the action of physical exogenous agents 
as the actinic light, the ultraviolet, and chemical rays as cosmetics, foods, gallates, toothpastes, dental brushes, metals, dental prosthesis and you plant. The incidence is bigger in the inferior lip and preferably in the feminine sex.

The liniment calcareous oil has demonstrated being of utility in the symptomatic treatment of the cheilitis for sharp contact. The present work looks for to evaluate the therapeutic action of the liniment calcareous oil in the treatment of the cheilitis for chronic contact.

\section{Keywords}

Inflammation - lip - cheilitis - pharmacological treatment - cytology.

\section{Resumo}

Os queilite de contato crônicas são doenças com alta incidência, a sintomatologia é limitada, e seus prováveis motivos para as mudanças que merece ser servido.

O processo é uma reação inflamatória inespecífica à ação de agentes físicos exógenos, como a luz actínica, raios ultravioletas, e produtos químicos, como cosméticos, alimentos, galatos, creme dental, escovas de dentes, metal, dentaduras, e andares. $\mathrm{A}$ incidência é maior no lábio inferior $\mathrm{e}$ de preferência na fêmea.

O linimento óleo calcário tem se mostrado útil no tratamento sintomático da queilite de contato aguda. Este trabalho visa avaliar a ação terapêutica de linimento óleo calcário no tratamento da queilite de contato crônica.

\section{Palavras chave}

Inflamação - lábio - queilite - tratamento medicamentoso - Pap.

\section{Introducción}

Las inflamaciones de los labios revisten singular importancia dado la peculiaridad histológica de la zona de transición de la piel a la mucosa, identificada como semimucosa labial, bermellón o zona de transición de Klein, que se manifiesta desde el punto de vista terapéutico como un órgano con poca expresividad, en el sentido de que causas muy diversas pueden tener las mismas exteriorizaciones clínicas ${ }^{1,2}$.
Las queilitis son patologías cada vez más frecuentes debido al incremento exposición a la luz solar agregado a la disminución de la capa de ozono con el consiguiente aumento a las acciones de rayos ultravioletas, por el uso de cosméticos, alimentos, medicamentos en especial tópicos que contienen galatos, dentífricos, cepillos dentales, metales, prótesis dentales, plantas otras sustancias exógenos químicos o físico que determinan una incidencia mayor en personas jóvenes con localización en el labio inferior y preferentemente en el sexo femenino ${ }^{3,4}$.

Las queilipatías se clasifican clínicamente en agudas, subagudas o crónicas y circunscriptas a la semimucosa labial, se expresan mediante lesiones elementales caracterizadas por eczemas, escamas, ampollas, fisuras, erosiones superficiales que se pueden cubrir de costras y fácilmente se agregan infecciones generalmente de tipo micóticas ${ }^{5,6,7}$.

Las enfermedades inflamatorias de los labios, en particular la recurrencia de las queilitis por contacto inespecíficas plantea situaciones que merecen ser atendidas por los riesgos que implica la evolución hacia la cronicidad, que en algunos casos, pueden sufrir una transformación maligna $^{8,9}$.

El contacto de los labios con agentes irritantes externos genera una reacción inflamatoria producida por un mecanismo inespecífico o específico, manifestado como reacción alérgica generalmente. Es así que las queilitis de contacto surgen cuando a partir de una o más aplicaciones de un agente de origen externo dan inicio al proceso inflamatorio ${ }^{10,11,12}$.

Estos cambios histológicos deben ser monitoreados y la mejor manera de efectuar el proceso es a través de los estudios anátomopatológicos ${ }^{13,}$ ${ }^{14}$ específicamente por medio citología exfoliativa tanto de la evolución de la enfermedad como del tratamiento ${ }^{15,16 .}$

Las queilitis de contacto a que se hace referencia en este estudio son las queilitis exfoliativas o descamativas que surgen como resultado de las exposiciones o bien de aplicaciones de agentes externos en la zona labial que ocasionan una respuesta inflamatoria mediada por un mecanismo irritativo primario ${ }^{17,} 18,19,20$.

La noxa que entra en contacto con la semimucosa labial es generalmente una sustancia de 
bajo peso molecular y no alergénica por sí misma, que causa irritación y se manifiesta como una reacción clínica eritematosa ${ }^{22,23,24}$.

Las queilitis por contacto descamativas tienen su manifestación inicial con una fase aguda del proceso inflamatorio caracterizado por síntomas como dolor, ardor o quemazón ${ }^{25,26 .}$

Los signos en los labios son el producto de la vasodilatación y de la congestión activa que confieren a los labios las características de edematosa, eritematosa y caliente. ${ }^{3 .}$ Luego se produce una diapédesis leucocitaria y una exudación plasmática producida por un exceso de líquido en el espacio intersticial ${ }^{27,28}$.

El cuadro clínico caracterizado inicialmente por calor y dolor provoca en muchos casos dificultades en la fonación y deglución. Posteriormente, se forman vesículas y ampollas que evolucionan hacia la formación de costras serosas o serohemáticas, que si son arrancadas pueden dejar fisuras y erosiones habilitando de este modo el asentamiento de infecciones secundarias ${ }^{29,30}$.

Las lesiones se localizan en la semimucosa, y aunque inicialmente surgen en la zona de contacto con el agente causal, luego se pueden extender a otras zonas vecinas. La sintomatología desaparece a los pocos días después de eliminar el factor causal, aunque en ciertos casos puede cursar hacia la cronicidad', 5, 31.

De persistir la causa, este puede interactuar con proteínas celulares de la piel y actuar como un complejo molecular induciendo un proceso de sensibilización en un período de tiempo de una a cuatro semanas al cabo de las cuales, una nueva exposición al agente causal, desencadenará una reacción alérgica, como las generadas por el contacto con materiales utilizados en las prótesis dentales en la que los labios forman parte de las estructuras anatómicas de preferencia para el asiento de esta patología ${ }^{32,33}$. En el caso de que el agente causal sea la irritación actínica pueden derivarse a cánceres labiales que en un $80 \%$ son de tipo espinocelular ${ }^{34}$.

El tratamiento de este tipo de queilitis, requiere como primera medida la identificación y la anulación del agente productor ${ }^{35}$. Para las erosiones y úlceras se pueden utilizar protectores como linimento óleo calcáreo, en todo caso una buena protección del área lesionada mediante sustancias emolientes que imiten la humectación natural es suficiente sin la necesidad de adicionar medicamentos con actividad intrínseca. En un trabajo previo se evaluó la efectividad de las formulaciones de linimento óleo calcáreo en pacientes con lesiones de hasta 48 hs de evolución. Los resultados estadísticos con muestras no paramétricas dan cuenta de una primacía cuantitativa de la formulación experimental. ${ }^{36}$. En casos más graves, corticoides tópicos como triamcinolona vehiculizado en un bioadhesivo. En el caso de reacción alérgica grave puede estar indicada la administración de corticoides por vía general ${ }^{37}$.

\section{Materiales y Método}

Es un estudio comparativo, prospectivo, sobre la utilización de linimento óleo calcáreo compuesto respecto del linimento óleo calcáreo simple elaborado según la Farmacopea Nacional Argentina. El estudio cuenta con la aprobación del Comité de Bioética de la Investigación de la Facultad de Odontología de la Universidad Nacional del Nordeste.

La muestra se conformó con pacientes de demanda espontánea que concurrieron a la Clínica Asistencial de la Cátedra de Clínica Estomatológica y con pacientes derivados de otras Cátedras Clínicas, del Servicio de Urgencia y del Hospital Odontológico. En todos aquellos pacientes con diagnóstico de queilitis por contacto se confeccionó la historia clínica diseñada para el presente estudio. Para que los pacientes puedan participar en el estudio se tuvieron en cuenta los siguientes criterios de inclusión:

- Pacientes de ambos sexos mayores de 15 años de edad.

- Pacientes con alteraciones en los labios con diagnóstico clínico de queilitis por contacto con más de 48 horas de evolución.

Y como criterios de exclusión a los pacientes que:

- No acordó los términos y condiciones del formulario de aceptación.

- La enfermedad diagnosticada clínicamente tenía menos de 48 hs de evolución.

- Pacientes menores de 15 años de edad.

- Pacientes que padecían enfermedades de alto riesgo médico, antecedentes de alergia o paciente con antecedentes de atopía.

- Pacientes que consumían sustancias adicti- 
vas, que alteraron el grado de cumplimiento del tratamiento.

- Inestabilidad domiciliaria.

Los pacientes luego de haber consentido por escrito participar en el estudio, mediante un formulario elaborado al efecto, fueron incorporados al estudio y se agruparon en forma aleatoria simple en dos grupos, el primero fue identificado como grupo control y el segundo como el grupo experimental.

El primer grupo recibió el tratamiento con una formulación simple de linimento de óleo calcáreo (L.O.C.f.s.) y el segundo grupo se trató con linimento de oleo calcáreo formulación objeto de estudio (L.O.C.f.o.e.).

Se realizó el registro de cada historia clínica con un número de orden creciente que permitió asignar por muestreo probabilístico aleatorio simple a dos grupos, uno corresponde a los que recibieron el tratamiento con L.O.C.f.s. y el otro grupo fue tratado con la L.O.C.f.o.e. esta última posee los componentes clásicos del linimento óleo calcáreo a los que se les adiciona manzanilla al 10\% por las propiedades emolientes y regenadoras epiteliales y vitamina $A$ al $1 \%$ por su acción queratoplástica.

Se consideró como criterio de salida a los pacientes que interrumpieron el tratamiento $o$ dejaron de asistir a los controles.

Previo al inicio de la terapéutica se eliminó la causa etiológica y se recolectaron muestras para un primer estudio de citología exfoliativa que se tomaron como parámetro histológico de base en este estudio comparativo y se implementó el tratamiento, con una posología de seis aplicaciones diarias.

El control y la evaluación del tratamiento se realizaron en varios tiempos operatorios a ambos grupos, correspondientes a los tres y siete días, que coincidió con la supresión de la medicación y la toma de muestras para un segundo y tercer estudio citológico respectivos.

Las muestras se tomaron por frotis convencionales y los extendidos fueron procesados en Servicio de Anatomía Patológica de la Facultad de Odontología, coloreados con la técnica de Papanicolaou (Hematoxilina, EA36, Orange), observados y evaluados con microscopio óptico a 40X; 100X y 400X.

Se registraron los datos observados en Pro- tocolos Sistematizados de Citología. Se utilizó un mismo observador calibrado que realizó el diagnóstico citológico especificando si hay inflamación (Dcl) o no hay inflamación (Dc2) y la intensidad de la misma en base al porcentaje de elementos inflamatorios observados.

En cada control se consignó el estado de la lesión, identificadas como leve mejoría, franca mejoría, cura completa o persistencia de la lesión de acuerdo a las modificaciones resultantes a la valoración de la sintomatología mediante una escala de Downie modificada y los signos mediante una escala milimetrada la superficie afectada.

\section{Resultados}

Se realizaron exámenes estomatológicos a 44 pacientes, 22 pacientes se incluyeron en el grupo de control que fueron tratados con L.O.C.f.s. y 22 pacientes se incluyeron en el grupo experimental que fueron tratados con L.O.C.f.o.e.

El primer estudio de citología exfoliativa (Cexl) previa a la administración de las formulaciones, coincidente con el tiempo 0 , dio como resultado que en los 44 casos se registró extendido inflamatorio, negativo para malignidad.

Al primer control correspondiente al tercer día, el total de pacientes del grupo control tratados con L.O.C.f.s demostraron un estado evolutivo de la lesión comprendido entre una leve mejoría en 14 casos y persistencia de la lesión en ocho casos. En el grupo experimental tratados con L.O.C.f.o.e, siete casos demostraron una leve mejoría y 15 casos una franca mejoría.

El segundo estudio de citología exfoliativa (Cex2) realizado en el primer control, arrojo el resultado de extendido inflamatorio en todos los pacientes de ambos grupos.

Al séptimo día, los pacientes del grupo control tratados con L.O.C.f.s. presentaron un estado de leve mejoría en 13 casos, además siete pacientes lograron un estado de franca mejoría y dos pacientes accedieron al estado de cura completa. Mientras en el grupo experimental tratados con L.O.C.f.o.e, siete pacientes mostraron un estado de franca mejoría y 15 pacientes un estado de cura completa.

El tercer estudio de citología exfoliativa (Cex3) realizada después de la administración de las formulaciones, dio como resultado que 
en los pacientes del grupo control tratados con L.O.C.f.s. se logró la cura completa en dos casos y persistencia de la lesión con presencia de extendido inflamatorio en 22 casos. En los pacientes grupo experimental tratados con L.O.C.f.o.e dio como resultado un estado de franca mejoría en siete pacientes y un estado de cura completa en 15 casos.

Se realizó el cálculo de la proporción de pacientes con presencia de inflamación y de los pacientes sin inflamación, para el grupo control (fig. I) y para el grupo experimental (fig. 2), en un segundo control coincidente con la Cex3.

No se detectaron reacciones adversas de medicamentos (RAM) en ninguno de los grupos de estudio.

Se contrastó la hipótesis de igualdad de proporciones de éxito en dos poblaciones, considerando éxito la categoría Dc2. Los valores $\mathrm{P}$ obtenidos para los grupos control y experimental fueron $\mathrm{pl}=0.09$ y $\mathrm{p} 2=0.68$ respectivamente. Se realizó la prueba basada en el estadístico de Fisher la cual arrojó un P-valor $=0.00006$. Concluyéndose que las diferencias son altamente significativas. Para relacionar estado de la lesión al tiempo 2 de ambos grupos se realizó una tabla
Grupo Control

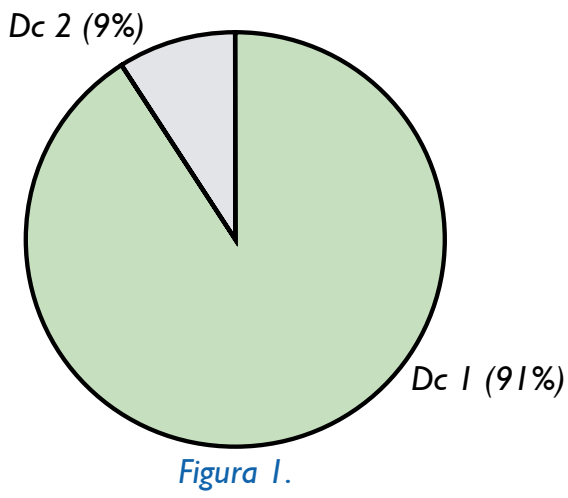

Grupo Control

Dc 2 (68\%)

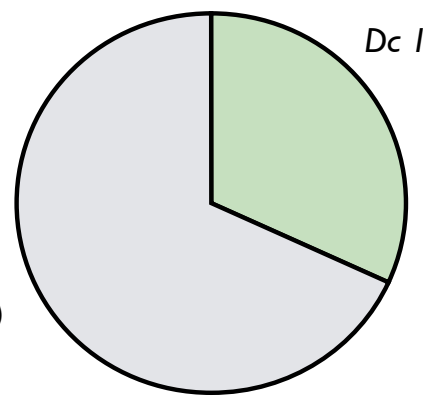

Figura 2.

Tabla I. Frecuencias absolutas en el control del tratamiento en el tiempo 2.

\begin{tabular}{l|c|c|c|c}
\hline & \multicolumn{3}{|c|}{ Tiempo 2 } & \\
\hline Grupo & Leve mejoría & Franca mejoría & Cura completa & Total \\
\hline Control & 13 & 7 & 2 & 22 \\
\hline Experimental & 0 & 7 & 15 & 22 \\
\hline Total & 13 & 14 & 17 & 44 \\
\hline
\end{tabular}

Tabla 2. Frecuencias relativas en el control del tratamiento en el tiempo 2.

\begin{tabular}{l|c|c|c|c}
\hline & \multicolumn{3}{|c|}{ Tiempo 2 } & \\
\hline Grupo & Leve mejoría & Franca mejoría & Cura completa & Total \\
\hline Control & 0.59 & 0.32 & 0.09 & 1.00 \\
\hline Experimental & 0.00 & 0.32 & 0.68 & 1.00 \\
\hline Total & 0.30 & 0.32 & 0.39 & 1.00 \\
\hline
\end{tabular}


de contingencia con frecuencias absolutas (tabla I) y otra tabla con frecuencias relativas por fila (tabla 2).

Estos resultados demuestran que para el grupo control sólo $9 \%$ tuvo una cura completa mientras que para el grupo experimental fue el $68 \%$. Y que para el grupo experimental el $100 \%$ tuvo una franca mejoría o cura completa mientras que el grupo control un $59 \%$ tuvo una leve mejoría.

\section{Discusión}

La revisión de las historias clínicas de los pacientes atendidos en este estudio, demuestran que las causas etiológicas en la generación de queilitis de contacto guardan similitud con los factores productores registrados en la bibliografía consultada y que la exposición de los labios a la acción de los agentes externos es la responsable de una respuesta inflamatoria que se traduce en síntomas y signos característicos como ardor, sensación de hinchazón, además de la aparición de escamas, fisuras y en algunos casos costras serosas o serohematicas. ${ }^{2,4,5,8}$.

Con posterioridad a la eliminación de los agentes externos detectados compatibles con las observadas en documentos consultados 17, 20,22, 23 y la aplicación de la formulación de linimento óleo calcáreo que ha demostrado ser efectiva en la remisión del cuadro clínico y en la consecuente recuperación de la salud labial, los resultados alcanzados en el presente estudio son análogos a los obtenidos en estudios previos realizados en queilitis por contacto agudas y tratados con la misma formulación óleo calcáreas. ${ }^{36}$.

Se registró bienestar y alivio de síntomas por la actividad protectora del linimento óleo calcáreo, sin la presencia de reacciones adversas. La formulación es de fácil uso por su aplicación tópica y carente de actividad intrínseca a diferencia de los corticosteroides vehiculizados en bioadhesivos. Teniendo como punto de partida los buenos resultados obtenidos y con el fin de mejorar la presentación de la formulación se ha previsto un nuevo trabajo de investigación, basado en la protección de la semimucosa labial lesionada mediante el uso sustancias oleo calcáreas con modificaciones en algunos de sus componentes, con el fin de mejorar la fragancia propia del aceite de lino.

\section{Conclusiones}

Es importante destacar que la valoración comparativa de las evoluciones de los grupos demuestra que ambas medicaciones reducen en grado variable la intensidad de los signos y la sintomatología.

Los resultados obtenidos dan cuenta de una importante supremacía de la formulación experimental, demostrada en la evolución de las lesiones a un estado de normalidad y la remisión completa de la sintomatología.

Las medicaciones ofrecen la posibilidad de una terapéutica tópica sin efectos adversos en pacientes con lesiones propias de queilitis irritativas por contacto con evoluciones de más de 48 horas.

Se recomienda el uso de la formulación de oleo calcáreo más vitamina $A$ al $I \%$ y manzanilla al $10 \%$ como sustituta de medicaciones de uso tópico en el tratamiento de las queilitis por contacto, por su bajo costo, fácil elaboración y fácil aplicación. 
REVISTA FACULTAD DE ODONTOLOGÍA

ISSN No 1668-7280 - Vol. VI No 2 - 2013
INVESTIGACION

Acción del óleo calcáreo en el tratamiento

de las queilitis por contacto crónicas.
I. Ceccotti, E. Sforza, R. "El Diagnóstico en Clínica Estomatológica”. Editorial Panamericana. Bs. As. Argentina. 2077. 300302.

2. Lascaris, G. Patologías de la Cavidad Bucal en Niños y Adolescentes. Ed. Actualidades Médico Odontológicas Latinoamérica, C. A. Caracas. Venezuela. 200I. 72-78.

3. Sapp, J. Wysocki, G. Patología Oral y Maxilofacial Contemporánea. Segunda Edición. Gráfica Hermanos Gómez. S. L. L. España. 2006. 278-279.

4. Regezi-Sciubba. Patología Bucal. Segunda Edición. Editorial Interamericana. México. 2006. 94-95.

5. Laskaris, G. Atlas de Enfermedades Orales. Primera Edición. Barcelona. España. 2005. I32-I33.

6. Aguas, S. Lanfranchi Tizeira, H. Lesiones premalignas o cancerizables de la cavidad oral. Revista de la Facultad de Odontología (UBA). Argentina. 2004. Vol. 19. N 47. P 26-28.

7. Velazco, G. Weinhold, E. Alfonso, R. Cova Natera, J. Clinical associations of type IV hypersensitivity in a patient under prostetic treatment. Rev. Odontológica de los Andes. 2006. Vol I $N^{\circ}$ 2. 53-60.

8. Mallo Pérez, L. Díaz Donado, C. Alergia de contacto intraoral a los materiales de uso odontoestomatológico. Una revisión crítica. Med Oral. 2003; 8: 334-47.

9. Velazco, G. Weinhold, E. Alfonso, R. Cova Natera, J. Clinical associations of type IV hypersensitivity in a patient under prostetic treatment. Rev Odontológica de los Andes. 2006. Vol I $N^{\circ}$ 2. 53-60.

10. Nettis, E. Colanardi, M. Ferrannini, A. Tursi, A. Reported latex allergy in dental patients. Oral Surg Oral Med Oral Pathol Oral Radiol Endod. 2002 Feb; 93 (2): I44-8.

II. Tanaka, B. Inaba, Y. Nakagawa M. Suzuki, K. Matsunaga, K. A case of contact cheilitis due to glyceryl isostearate. J Environ Dermatol Cutan Allergol 2009: 3: 163-169.

12. Lim, S. Goh, C. Epidemiology of eczematous cheilitis at a tertiary dermatological referral centre in Singapore. Contact Dermatitis 2000: 43: 322-326.

13. Vallejos, A. Manual de Introducción a la Anatomía Patológica. UNNE. Facultad de Odontología. Cátedra de Anatomía Patológica. 2007.

14. Diniz Freitas, M. García García, A. Crespo-Abelleira, A Martins Carneiro, J. Gándara Rey, J. Aplicaciones de la citología exfoliativa en el diagnóstico del cáncer oral. Med Oral 2004; 9: 355-36I.

15. Downer, M. Moles, D. Palmer, S. Speight, P. A systematic review of measures of effectiveness in screening for oral cancer and precancer. Oral Oncol 2006; 42 (6): 55I-560.

16. Solomon, D. Davey, D. Kurman, R. Moriarty, A. et al. The 200I Bethesda System. JAMA, 287: 21 14, 2002.

17. Francalaci, S. Sertoli, A. Giorgini, S. Pigatto, P. et al. Multicentre study of allergic contact cheilitis from toothpastes. Contact Dermatitis. 2000. 43 (4): 216-22.

18. Zoli, V. Silvani, S. Vincenzi, C. Tosti A. Allergic contact cheilitis. Contact Dermatitis. 2006: 54: 296-297.

19. Hauschild, A. Lischner, S. Lange-Asschenfeldt, B. Egberts, F. Treatment of actinic cheilitis using photodynamic therapy with methylaminolevulinate: report of three cases. Dermatol Surg. 2005; 31: 1344-8.

20. Quartier, S. Garmyn, M. Becart, S. Goossens, A. Allergic con- tact dermatitis to copolymers in cosmetics-case report and review of the literature. Contact Dermatitis 2006: 55: 257-267.

2I. Zug, K. Kornik, R, Belsito, D. DeLEO, V. et al; North American Contact Dermatitis Group. Patch-testing North American lip dermatitis patients: data from the North American Contact Dermatitis Group, 200 I to 2004. Dermatitis. 2008 Jul-Aug; 19 (4): 202-8.

22. Paulsen, E. Contact sensitization from Compositae-containing herbal remedies and cosmetics. Contact Dermatitis. 2002: 47: 189-198.

23. Farina, R. Andreotti, C. Chronic relapsing allergic contact cheilitis from a toothpaste. A case report. Minerva Stomatol. 2007 Mar; 56 (3): 145-52.

24. Sowa, J Suzuki, K. Tsuruta, K. Akamatsu, H. Matsunaga, K. Allergic contact dermatitis from propylene glycol ricinoleate in a lipstick. Contact Dermatitis 2003: 48: 228-229.

25. Pereira, F. Hatia, M. Cardoso, J. Systemic contact dermatitis from diallyl disulfide. Contact Dermatitis 2002: 46: 124.

26. Akasya-Hillenbrand, E. Özkaya, E. Patch test results in 542 patients with suspected contact dermatitis in Turkey. Contact Dermatitis 2002; 46: 17-23.

27. Strauss, R. Orton, D. Allergic contact cheilitis in the United Kingdom: a retrospective study. Am J Contact Dermat. 2003 Jun; I4 (2): $75-7$.

28. Torgerson, R. Davis, M. Bruce, A. Farmer, S. Rogers, R. 3rd. Contact allergy in oral disease. J Am Acad Dermatol. 2007 Aug; 57 (2): 3 I5-2I. Epub 2007 May 25.

29. Rioboo Crespo, M. Planells Del Pozo, P. Rioboo García, R Epidemiology of the most common oral mucosal diseases in children. Med Oral Patol Oral Cir Bucal. 2005; 10 (5): 376-387.

30. López Sánchez, A. González Romero, E. Conceptos Básicos de odontoestomatología para el médico de atención primaria. Ed Masson 200I. |3|- 132

3I. Berking, C. Herzinger, T. Flaig, M. Brenner, M. Borelli, C. Degitz, K. The efficacy of photodynamic therapy in actinic cheilitis of the lower lip: a prospective study of 15 patients. Dermatol Surg. 2007; 33: 825-30.

32. Gawkrodger, D. Investigation of reactions to dental materials. Br J Dermatol. 2005 Sep; 153 (3): 479-85.

33. Khamaysi, Z. Bergman, R. Weltfriend, S. Positive patch test reactions to allergens of the dental series and the relation to the clinical presentations. Contact Dermatitis. 2006 Oct; 55 (4): 216-8.

34. Kodama, M. Watanabe, D. Akita, Y. Yasuhiko, T. Matsaumoto, Y. Photodynamic therapy for the treatment of actinic cheilitis. Photodermatol Photoimmunol Photomed. 2007;23: 209-10.

35. García López, E. Blanco Ruiz, A., Rodríguez García, L. Reyes Fundora, D. Sotres Vázquez, J. Queilitis: Revisión bibliográfica. Rev Cubana Estomatol [revista en la Internet]. 2004 Ago [citado 2012 Jun 28]; 4 I (2):

36. Fernández, V. Rosende, R. Ortiz Barreto, E. Análisis comparativo de dos medicaciones en el tratamiento de las queilitis". Revista de la Facultad de Odontología de la Universidad Nacional del Nordeste. Volumen II. N². Año 2009. Argentina. Pág. 20-23.

37. Lee, P. Elsaie, M. Jacob, S. Allergic contact dermatitis in children: common allergens and treatment: a review. Curr Opin Pediatr 2009; 2I: 49I-8. 